\title{
The essential oil and its main constituents of Origanum syriacum ssp. sinaicum grown wild in Saint Katherine Protectorate, South Sinai, Egypt
}

\author{
A. A. Badawy ${ }^{1,}{ }^{*}$, M. A. El-mohandes ${ }^{1}$, A. M. Algharib ${ }^{1}$, B. E. Hatab ${ }^{2}$ and E. A. Omer ${ }^{3}$ \\ ${ }^{1}$ Department of Environment and Bio-Agriculture, Faculty of Agriculture, Al-Azhar University, Cairo, Egypt \\ ${ }^{2}$ Egyptian Environmental Affairs Agency (EEAA), Ministry of Environment, Cairo, Egypt \\ ${ }^{3}$ Department of Medicinal and Aromatic Plants Research, National research Centre, Dokki, Cairo, Egypt
}

*Corresponding author email: anas_abdelaziz2020@azhar.edu.eg (A. Badawy)

\begin{abstract}
This work aims to study the essential oil and its main constituents of $O$. syriacum grown wild in 22 locations in Saint Katherine Protectorate. Origanum syriacum ssp. sinaicum plants collected during the summer season of 2014 from 22 locations in Saint Katherine Protectorate (SPK), South Sinai Egypt. Depending on location length, each location was divided into 2 stands to reach a total of 44 stands. Twenty compounds were identified as the main constituents of the essential oil which accounted for $c a 97 \%$ from the total compounds of the essential oil. Carvacrol was the major constituent in all collected plants and ranged from $74.2 \%$ to $92.68 \%$ from the total compounds of the essential oil. Where, P-cymene was identified in the essential oil of all studied stands and ranged for $0.98 \%$ to $6.23 \%$. The same was observed for $\gamma$-terpinene that was identified in the essential oil of all plants in the 44 stands and accounted for $1.37 \%$ as minimum percent up to $7.4 \%$ as maximum percent from the total compounds. The oxygenated compounds in the essential oil of $O$. syriacum were identified as carvacrol, terpienol-4, linalool, borneol, thymol, eugenol, and long pinenee poxid with relative percentage from $76.6 \%$ to $94.2 \%$. The nonoxygenated compounds hydrocarbons ranged from $3.7 \%$ to $18.4 \%$ in which $P$-cymene and $\mathrm{y}$-terpinene were the main non-oxygenated compounds. The relations between the essential oil\%, as well as between carvacrol\% in the essential oil in the different stands and calcium content, $\mathrm{Cl}$ and $\mathrm{Na}$ in the soil of the different stands were studied. In conclusion, the essential oil \% of O. syriacum ssp. sinaicum and its main constituents changed according to the altitude and soil contents.
\end{abstract}

Keywords: Origanum syriacum; essential oil; carvacrol; GC-MS; Saint Katherine.

\section{INTRODUCTION}

Medicinal plants and culinary herbs have long been known as one of the basis of traditional medicine in many countries such as Egypt. Some important medicinal wild plants suffered from unwise human manipulation which resulted in the extinction of some species. Projects to recover the genetic diversity of wild plants were carried out in Egypt accompanied with scientific research to evaluate their use based on traditional medicine in Bedouin communities. Indeed, there are a great number of rural jobs dependent on this sector (ViudaMartos et al., 2010, 2011). Origanum syriacum. ssp. sinaicum (Boiss.) family Labiatae is a rare perennial wild herb endemic to Sinai. $O$. syriacum is represented by the following three varieties: ssp. syriacum, distribution Palestinian, Jordon, Syria; ssp. bevanii, Turkey, Syria, Lebanon, Cyprus; ssp. sinaicum is cultivated in Northern Sinai in Egypt and Palestinian. The plant was described by Ietswaat in his treatise on the taxonomy of the genus Origanum (Başer et al., 2003). The herbal parts of Origanum species were used by local people for herbal tea and as a spice in soups, salads, olives and meats. The remaining water after the plant water distillation is used orally to reduce cholesterol and glucose levels as well as to treat cancer (Kizil et al., 2008). It is also used as stimulant, analgesic, antitussive, expectorant, sedative, anti-parasitic and anti-helminthic (Dundar et al., 2008). Tepe et al., (2004) found that the plant's essential oil of $O$. syriacum could be used as a natural preservative in the food industry. Kamel et al., (2001), reported that Origanum plants have many different uses such as powerful disinfectants, flavoring agents, in perfumes and in scenting soaps. Some studies suggested that Origanum may have antioxidant effects which may be due to the oil components of some of the active substances, such as phenols carvacrol and thymol (Baricevic and Bartol, 2002). On the other hand, Sokovic et al., (2007), found that Origanum plant is a good source of antimicrobial compounds. Kalemba and Kunicka, (2003), indicated that this plant was used in ancient times as a natural food preservative and as a flavoring agent. Owlia et al., (2009), found that this plant was successful treatment of infectious diseases. Tackholm (1974) recorded that the Origanum syriacum plant was growing wild in the Sinai Desert of Egypt. Carvacrol and/or thymol represent the major constituents of Origanum essential oil 
(Sarer et al. 1982, Skoula and Harborne, 2002, Loizzo et al., 2009; Zein et al., 2011). The oil quality is determined by its composition, which varies with genotype, plant development, climate, and soil type (Russo et al., 1998; Baydar et al., 2004). Elgindy et al. (2015) identified 46 compounds in the essential oil of Origanum syriacum plants which collected from Sinai, Egypt. They reported that Carvacrol was dominated in cultivated plants, while thymol, $\gamma$-terpinene, linalool and 4-terpineol were dominated in the wild plants. Shalaby et al., (2011), reported the high potentials of O. syriacum var. sinaicum plant as new crop in Egypt. Therefore, this work aims to study the essential oil and its main constituents of $O$. syriacum grown wild in 22 locations in Saint Katherine Protectorate, South Sinai, Egypt.

\section{MATERIALS AND METHODS}

\section{Plant Materials:}

Origanum syriacum plants were collected from 22 locations in Saint Katherine Protectorate (SKP), South Sinai, Egypt which includes Frosh, Wadis and Gorge systems during summer season (June) of 2014. Depending on location length, each location was divided into stands in total 44 stands. The geographical (latitudes, longitudes and alludes) data of the different studied stands are shown in Table (1). The plant samples were collected from the aerial parts of growing plants in the same time from all the stands of the study. The plant samples were separately air dried in the shades till the weight was constant and then kept in paper bags and kept in desiccators till essential oil extraction.

\section{Extraction of essential oil (EO):}

The essential oil (EO) was extracted from the air-dried areal parts of $O$. syriacum by hydrodistillation using a Clevenger type apparatus for $3 \mathrm{~h}$ according to Guenther (1961). The oily layer obtained on top of the aqueous distillate was separated and dried with anhydrous sodium sulfate. The extracted EOs were kept in sealed air-tight glass vials and covered with aluminum foil at $4 \circ \mathrm{C}$ until GC-MS analysis.

\section{Gas Chromatography-Mass Spectrometry (GC-MS):}

The GC-MS analysis of the essential oil was carried out using Gas Chromatography-Mass Spectrometry instrument stands at the Department of Medicinal and Aromatic Plants Research, National Research Center, Egypt. Most of the compounds were identified using mass spectra of authentic chemicals (Wiley spectral library collection and NSIT library 2000). Further identifications were carried out using the MS literature data (Adams, 2004).

\section{Soil physical analysis:}

The physical properties of the soil samples that collected from all stands were analyzed to determine water, sand, silt and clay contents according to Piper (1950). From these results the soil texture of each stand was determined as shown in Table (2).

\section{Soil chemical analysis:}

Some chemical parameters i.e. $\mathrm{pH}, \mathrm{EC}$, organic matter, $\mathrm{CaCO} 3$, some cations $(\mathrm{Ca}, \mathrm{Mg}$, $\mathrm{Na}$ and $\mathrm{K})$ and some anions $\left(\mathrm{HCO}_{3}, \mathrm{Cl}\right.$ and $\left.\mathrm{SO}_{4}\right)$ were determined in the beasts of the soil samples according to Jackson (1967) and Allen et al. (1976). Oxidizable organic carbon (as indication of the total organic matter) was determined using Walkely and Black rapid titration method as described by Black (1965). The results of these parameters are shown in Table (3).

\section{Statistical Analyses:}

Data were statistically analyzed using Oneway ANOVA and Post hoc-LSD tests (the least significant difference) (SPSS Inc., 2009) at 0.05, 0.01 and 0.001 level of probability (Snedecor andCochran, 1982).

\section{RESULTS AND DISCUSSION}

Data in Table (4) represents the essential oil percentage of the aerial parts of Origanum syriacum ssp. sinaicum plants collected from the different stands which ranged from $2.25 \%$ in stand No.31 to $6.75 \%$ in stand No.1. It is clear that the minimum essential oil \% was observed in the lowest altitude (1429), while the maximum \% (6.75\% and 6.5\%) were determined in plants of the highest altitudes (2002 and 2016, respectively). Figs. (1a), (2a) and (3a) indicate the relation between essential oil \% of Origanum syriacum ssp. sinaicum plants in the different stands and calcium, $\mathrm{Cl}$ and $\mathrm{Na}$ contents in the soil of the different stands. It is clear that the essential oil decreased with increasing calcium, $\mathrm{Cl}$ and $\mathrm{Na}$ contents. The opposite trend was observed with $\mathrm{CaCO}_{3}$, in which the essential oil $\%$ increased with increasing $\mathrm{CaCozcontent} \mathrm{of}$ the soil (Fig., 4b). The relation between essential oil percent of $O$. syriacum plant sand electrical conductivity (EC) in the different stands is presented in Fig. (5a). The results indicated that essential oil \%decreased with increasing level of EC. The main oil compounds are shown in Table (5). Twenty compounds were identified as the main constituents of the essential oil and 
accounted for $\mathrm{ca} 97 \%$ from the total compounds of the oil. Carvacrol was found to be the major compound in all collected plants from different stands and ranged from $74.2 \%$ (stand 38 ) to $92.68 \%$ (stand10) from the total compounds of the essential oil. $P$-cymene was identified in the essential oil of all studied stands and followed like carvacrol in the relative percentages, since it ranged for $0.98 \%$ (stand 10 ) to $6.23 \%$ (stand 38). The same was observed for $\gamma$-terpinene which was identified in the essential oil of all plants in 44 stands and accounted for $1.37 \%$ (stand 6) as minimum percent to7.4\% (stand 38) as maximum percent from the total compounds. The Oxygenated compounds in the essential oil of $O$. syriacum ssp. sinaicum were identified as Carvacrol, Terpienol $-4, \alpha$ Terpineol,Linalool, Borneol, Thymol, Euginol and Long pineneepoxidl. They accounted from $76.6 \%$ (stand 38 ) to $94.2 \%$ (stand 10 ), while the non-oxygenated compounds ranged from 3.7\% (stand 10) to $18.4 \%$ (stand 38). Thymol percentage failed to reach $1 \%$ in the essential oil of all $O$. syriacum ssp. sinaicum in all stands, which ranged from $0.0 \%$ (stands 2, 18, 19, 33, 37 ) to $0.19 \%$ (stand 11 ). The maximum values of carvacrol (92.68., 91.74, and 91.41) were found in plants at the stands of Shak Musa 1, Wadi al-shak1 and Farsh Al-romana, respectively as shown in Table (5). While, the lowest percentage of carvacrol values (74.21, 79.68 and 79.86.) were found in stands at Wadi elarbain1, Taupq, and Seleebat, respectively.

Carvacrol \% in the essential oil increased with increasing soil calcium content, $\mathrm{Cl}$ and $\mathrm{Na}$ in the different stands to reach its maximum values with $8-12 \mathrm{meq}$, 60meq/and $175 \mathrm{pp}$, respectively as shown in Figs. (1b, 2b, 3b). On the other hand, carvacrol percent decreased with increasing $\mathrm{CaCO}_{3}$ in the soil (Fig., 4b). Carvacrol increased with increasing EC values up to 3 then tended to decrease (Fig 5b). The essential oils composition depends on many factors such as climate, geographical location and vegetative stage (Abu Lafi et al., 2007, 2008; Baser et al., 2003and Lukas et al., 2009). Baydar et al., (2004), concluded that oil quality is determined by its composition, which varies with genotype, plant development, climate, and soil type. Sangwan et al., (2001), reported that, there are many factors influence the essential oil composition such as plant ontogeny, site of oil production, photosynthesis, light quality, seasonal and climatic variations, nutritional relationships, plant growth regulators, plant density, moisture, salinity, temperature and harvesting methods, seasonal and climate conditions such as temperature and rainfall, and thus the seasonal variation of the main essential oil components have great impact. Skoula and Harborne (2002), indicated that the essential oil of Origanum is contains of carvacrol and/or thymol as major components, followed by $\gamma$-terpinene, p-cymene, linalool, terpinen-4ol and sabinene hydrate. Russo et al., (1998), found another chemo type between thymol and carvacrol which contains a high content of $\gamma$ terpinene or p-cymene. Shalaby et al., (2011), reported that the major constituents in the essential oil of $O$. syriacum var. sinaicum were dominated by thymol, $\gamma$-terpinene and $p$ cymene. Some studies indicate that the main component of $O$. syriacum oil differs according to the growing season (Soliman et al., 2007), which found carvacrol was the major component in summer season while thymol was the major component in autumn. On the other hand, Toncer et al. (2010) found that, the carvacrol levels varied from $0.73-8.9 \%$, peaked in August (8.9\%) and July (8.8\%) and was low in January $(0.73 \%)$. Also, some components such as $\alpha$-pinene and $\beta$-caryophyllene decreased in winter months however terpinen4-ol showed the opposite trend and increased in winter. Zein et al. (2011), also noticed a difference in the components of essential oil according to the different growing season, he reported that the thymol and carvacrol increased progressively from February to April, then, decreased in May. Our previous study shows comparative results, where the poor content of thymol and carvacrol in February and March was accompanied by an increased rate of $\gamma$-terpinene and p-cymene, which they are the precursors of thymol and carvacrol. This finding may help researchers and farmers about the optimal harvesting time, allowing yielding oil with high content of thymol and carvacrol. The optimal harvesting time was in April just before flowering. In this study, thymo quinone was detected as a trace amount only in samples harvested in February. This promising anticancer molecule was found in essential oil of O. syriacum in previous studies. Thymol, pcymene, $\alpha$-terpinene, $\gamma$-terpinene, carvacrol were found to be the major components in the oil, since monoterpenes, thymol, $p$-cymene and $\gamma$-terpinene, are biosynthetically related (Muller-Riebau et al., 1997). But the quantitative composition of the essential oil was changed during the 11-month sampling period. Thymol $(25.3 \%)$ was the major component in summer and autumn plants, while $p$-cymene was a major component in the winter oils (Toncer et al., 2010). 


\section{CONCLUSION}

Origanum syriacum ssp. sinaicum grown wild in Saint Kathrin Protectorate, South Sinai, Egypt is a Carvacrol chemo type since the major component in its essential oil was carvacrol. The essential oil \% of O. syriacum ssp. sinaicum and its main constituents changed according to the altitude and soil contents. No considerable differences were observed for the effect of soil texture neither on essential oil \% nor on carvacrol \%.

\section{REFERENCES}

Abu Lafi, O.I., Dewik, H., Qabajah, M., Imam, A., Dembitsky, V., Hanus, L.O., 2007. Natural compounds of Palestine flora. Comparison analysis by static headspace and steam distillation GC-MS of semivolatile secondary metabolites from leaves of cultivated Palestinian Majoranasyriaca. Biomed. Pap. Med. Fac. Univ. Palacky Olomouc Czech Repub., 151, 21-29.

Allen, S.E., Grimshaw, H.M., Parkinson, J.A., Quarmby, C., Roberts, J.D., 1976. Chemical Analysis of Ecological Materials. Edited by S.B. Chapman. Blackwell Sci. Publ., Oxford. pp-166411.

Baricevic, D. Bartol, T., 2002. The biological / pharmacological activity of the Origanum genus. In " Oregano: the genera Origanum and Lippia". Taylor \& Francis, (HAS Library), London.

Baser, H., Kürkçüoglu, M., Demirci, B., Özek, T. 2003. The esse-tial oil of Origanum Syriacum, ssp.Sinacum (Bioss) Iets wart. Flavour and Fragrance Journal. John Wiley \& Sons, Chichester, UK, 18 (2), 98-99.

Baydar, H., Sagdis, O., Ozkan, G., Karadogan, T., 2004. Antibacterial activity and composition of essential oils from Origanum, Thymbra and Satureja species with commercial importance in Turkey. Food Control 15, 169-172.

Black, C.A., 1965. Methods of Soil Analysis. Am. Soc. Agron. pp.771-1572.

Blaschek, W., Hänsel, R., Keller, K., Reichling, J., Rimpler, H., \& Schneider, G. (Eds.). (2013). Hagers Handbuch der Pharmazeutischen Praxis: Folgeband 2: Drogen AK. SpringerVerlag.

Dundar, E, Olgun E.G., Isiksoy, S., Kurkcuoglu, M., Baser, K.H.C., Bal, C., 2008. The effects of intrarectal and intra-peritoneal application of Origanum onites L. essential oil on 2,4,6trinitrobenzenesulfonic acid-induced colitis in the rat. Exp. Toxicol. Pathol., 59, 399-408.

El-Gendy, R., Kirkham, J., Newby, P.J., Mohanram, Y., Boccaccini, A.R., Yang, X.B., 2015. Investigating the vascularization of tissueengineered bone constructs using dental pulp cells and 4555 Bioglass (R) Scaffolds. Tissue Eng. A, 21, 2034-2043.

Guenther, E., 1961. The Essential Oils. VI. New York: Van Nostrand p. 353.

Blaschek, W., Hänsel, R., Keller, K., Reichling, J., Rimpler, H., \& Schneider, G. (Eds.). (2013). Hagers Handbuch der Pharmazeutischen Praxis: Folgeband 2: Drogen AK. SpringerVerlag.

Ibrahim, L., Karaky, M., Ayoub, P., El Ajouz, N., Ibrahim, S., 2012. Chemical composition and antimicrobial activities of essential oil and its components from Lebanese Origanum syriacum L. J. Essent. Oil Res., 24 (4), 339-345.

Jackson, M.L. 1967. Soil Chemical AnalysisAdvanced Course. Washington Department of Soil Sciences, pp.498

Kalemba, D., Kunicka, A., 2003. Antibacterial and antifungal properties of essential oils. Curr. Med. Chem. 10, 813-829.

Kamel, M.S., Assaf, M.H., Hasanean, H.A., Ohtani, K., Kasai, R., Yamasaki, K., 2001. Monoterpene glucosides from Origanum syriacum. Phytochemistry 58, 1149-1152.

Kizil, S., Ipek, A., Arslan, N., Khawar, K.M., 2008. Effect of different developing stages on some agronomical characteristics and essential oil composition of oregano (Origanum onites). New Zealand J. Crop Hor. Sci. 36 (1), 71-76.

Loizzo, M., Menichini, F., Conforti, F., Tundis, R., Bonesi, M., Saab, A., Statti, G., de Cindio, B., Houghton, P., Menichini, F., Frega, N. 2009 Chemical analysis, antioxidant, antiinflammatory and anticholinesterase activities of Origanum ehrenbergii Boiss and Origanum syriacum L. essential oils. Food Chem. 117, 174180.

Lukas, B., Schmiderer, C., Franz, C.H., Novak, J., 2009. Composition of essential oil compounds from different Syrian populations of Origanum syriacum L. (Lamiaceae). J. Agric. Food Chem., 57, 1362- 1365

Muller-Riebau, F., Berger, B., Yegen, O., Cakir, C., 1997. Seasonal variations in the chemical compositions of essential oils of selected aromatic plants growing wild in Turkey. J. Agric. Food Chem., 45, 4821-4825

Owlia, P., Saderi, H., Rasooli, I., Sefidkon, F., 2009. Antimicrobial characteristics of some herbal oils on Pseudomonas aeruginosa with special reference to their chemical compositions. Ind. J. Pharm. Res., 8 (2), 107-114.

Piper, C.S., 1950. Soil and Plant Analysis, Univ. Adelaide press. Australia.

Russo, M., Galletti, G.C., Bocchini, P., Carnacini, A., 1998. Essential oil chemical composition of wild populations of Italian Oregano spice (Origanum vulgare ssp. hirtum (Link) letswaart): a preliminary evaluation of their use in 
chemotaxonomy by cluster analysis 1 . Inflorescences. J. Agric. Food Chem. 46, 37413746.

Sangwan, N.S., Farooqi, A.H.A., Shabih, F., Sangwan, R.S., 2001. Regulation of essential oil production in plants, Plant Growth Regul., 34, 3-21.

Sarihan EO, Ipek A, Arslan N, Gürbüz B., 2006. The effects of different row spacing and intra-row spacing on yield and yield components of Oregano (Origanum vulgare ssp. hirtum), Tarım Bilim. Derg., 12 (3), 246-251.

Sarer, E., Scheffer, J.J.C., Svedsen, A.B., 1982. Monoterpenes in the essential oil of Origanum majorana. Planta Med., 46, 236-23.

Shalaby, A.S., Elhefnawy, N., Ghanem, K., Algharib, A.M., 2011. Agronomic and chemical comparison between Origanum vulgare ssp. hirtum and the cultivated plants of O. syriacum ssp. sinaicum. J. Essent. Oil Bearing Plants., 14 (4), 463-471

Skoula, M., Harborne, J.B., 2002. Taxonomy and chemistry. In: Kintzios, S.E., Ed. Oregano: The Genera Origanum and Lippia. Medicinal and
Aromatic Plants-industrial Profiles, Taylor \& Francis CRC Press, USA, pp 67-108.

Snedecor, G.M., Cochran, W.G., 1982. Statistical methods-7th edition, lowa state Univ., Press, Ames., lowa, USA., pp. 325- 330.

Sokovic, M., Marin, P.D., Brkic, D., van Griensven, L.J.L.D., 2007. Chemical composition and antibacterial activity of essential oils often aromatic plants against human pathogenic bacteria. Food 1, 220-226.

Soliman, F., Yousif, M., Zaghloul, S., Okba, M., ElSayed, E., El-Sayed, E., 2007. Seasonal variation in the essential oil composition of Origanum syriacum L. ssp. Sinaicum greuter and burdet; evaluation of its tocolytic activity, Egypt. J. Biomed. Sci. 23 (1), 121-134.

Täckholm, V., 1974. Student's Flora of Egypt. Cairo University Press. Cairo, Egypt, pp. 888.

Tepe, B., Daferera, D., Sokmen, M., Polissiou, M., Sokmen, A.Q., 2004. The in vitro antioxidant and antimicrobial activities of the essential oil and various extracts of Origanum syriacum L. varbevanii. J. Sci Food Agric., 84: 1389-1396. 
Table 1. The geographical data (latitudes, longitudes and alludes) of different studied stands in SPK, south Sinai Egypt during June of 2014

\begin{tabular}{|c|c|c|c|c|c|c|}
\hline $\begin{array}{l}\text { No. of } \\
\text { location }\end{array}$ & $\begin{array}{l}\text { Location } \\
\text { name }\end{array}$ & $\begin{array}{l}\text { Stands } \\
\text { No. }\end{array}$ & $\begin{array}{l}\text { Stands } \\
\text { name }\end{array}$ & E & $\mathrm{N}$ & Alt \\
\hline \multirow{5}{*}{1} & \multirow{5}{*}{$\begin{array}{l}\text { Frosh jabal } \\
\text { mousa }\end{array}$} & 1 & $\begin{array}{l}\text { Farsh al- } \\
\text { lozaa-1 }\end{array}$ & 33.97037 & 28.54838 & 2002 \\
\hline & & 2 & $\begin{array}{l}\text { Farsh al- } \\
\text { lozaa-2 }\end{array}$ & 33:96912 & 28.54906 & 2016 \\
\hline & & 3 & $\begin{array}{l}\text { Farsh al- } \\
\text { lozaa-3 }\end{array}$ & 33.97092 & 28.54835 & 1995 \\
\hline & & 4 & $\begin{array}{l}\text { Farsh al- } \\
\text { lozaa-4 }\end{array}$ & 33.97164 & 28.54843 & 1992 \\
\hline & & 5 & $\begin{array}{l}\text { Farsh al- } \\
\text { sofsafa }\end{array}$ & 33.96465 & 28.55447 & 1993 \\
\hline \multirow{2}{*}{2} & \multirow{2}{*}{$\begin{array}{l}\text { Wadi } \\
\text { egebal }\end{array}$} & 6 & $\begin{array}{l}\text { Farsh al- } \\
\text { romana-1 }\end{array}$ & 33.8826 & 28.35989 & 1801 \\
\hline & & 7 & $\begin{array}{l}\text { Farsh al- } \\
\text { romana-2 }\end{array}$ & 33.88369 & 28.53947 & 1801 \\
\hline \multirow{3}{*}{3} & \multirow{3}{*}{$\begin{array}{l}\text { Shak } \\
\text { graginiah }\end{array}$} & 8 & Graginiah-1 & 33.9695 & 28.52221 & 1980 \\
\hline & & 9 & Graginiah-2 & 33.97019 & 28.5235 & 1890 \\
\hline & & 10 & Graginiah-3 & 33.96853 & 28.51985 & 1857 \\
\hline \multirow{2}{*}{4} & \multirow{2}{*}{$\begin{array}{l}\text { Shak } \\
\text { mousa }\end{array}$} & 11 & $\begin{array}{l}\begin{array}{l}\text { Shak } \\
\text { mousa-1 }\end{array} \\
\end{array}$ & 33.96383 & 28.53001 & 1961 \\
\hline & & 12 & $\begin{array}{l}\text { Shak } \\
\text { mousa-2 }\end{array}$ & 33.96734 & 28.53342 & 1976 \\
\hline \multirow{3}{*}{5} & \multirow{3}{*}{$\begin{array}{l}\text { Wadi al- } \\
\text { arbain }\end{array}$} & 13 & $\begin{array}{l}\text { Wadi al- } \\
\text { arbain -1 }\end{array}$ & 33.96139 & 28. 53936 & 1726 \\
\hline & & 14 & $\begin{array}{l}\text { Wadi al- } \\
\text { arbain-2 }\end{array}$ & 33.96139 & 28.554026 & 1720 \\
\hline & & 15 & $\begin{array}{l}\text { Wadi al- } \\
\text { arbain-3 }\end{array}$ & 33.85895 & 28.54073 & 1731 \\
\hline \multirow{3}{*}{6} & \multirow{3}{*}{$\begin{array}{l}\text { Wadi al- } \\
\text { shak }\end{array}$} & 16 & $\begin{array}{l}\text { Wadi al- } \\
\text { shak-1 }\end{array}$ & 33. 93365 & 28.53454 & 1855 \\
\hline & & 17 & $\begin{array}{l}\text { Wadi al- } \\
\text { shak-2 }\end{array}$ & 33.93315- & 28.5365 & 1844 \\
\hline & & 18 & $\begin{array}{l}\text { Wadi al- } \\
\text { shak-1 }\end{array}$ & 33. 93257 & 28.53860 & 1828 \\
\hline \multirow{3}{*}{7} & \multirow{3}{*}{ Wadi itlah } & 19 & $\begin{array}{l}\text { Wadi itlah- } \\
1\end{array}$ & 33.9226 & 28.58395 & 1429 \\
\hline & & 20 & $\begin{array}{l}\text { Wadi itlah- } \\
2\end{array}$ & $33: 92565$ & 28.57904 & 1429 \\
\hline & & 21 & $\begin{array}{l}\text { Wadi itlah- } \\
3\end{array}$ & 33.93108 & 28.57148 & 1468 \\
\hline \multirow[t]{3}{*}{8} & \multirow{3}{*}{$\begin{array}{l}\text { Maen al- } \\
\text { raian }\end{array}$} & 22 & $\begin{array}{l}\text { Maen al- } \\
\text { raian-1 }\end{array}$ & 33.88637 & 28.53771 & 1811 \\
\hline & & 23 & $\begin{array}{l}\text { Maen al- } \\
\text { raian-2 }\end{array}$ & 33.84194 & 28.54008 & 1834 \\
\hline & & 24 & $\begin{array}{l}\text { Maen al- } \\
\text { raian-3 }\end{array}$ & 33.89539 & 28.5423 & 1861 \\
\hline 9 & $\begin{array}{l}\text { Abu } \\
\text { kasaba }\end{array}$ & 25 & $\begin{array}{l}\text { Abu } \\
\text { kasaba-1 }\end{array}$ & 33.89073 & 28.52291 & 1854 \\
\hline
\end{tabular}




\begin{tabular}{|c|c|c|c|c|c|c|}
\hline $\begin{array}{l}\text { No. of } \\
\text { location }\end{array}$ & $\begin{array}{l}\text { Location } \\
\text { name }\end{array}$ & $\begin{array}{l}\text { Stands } \\
\text { No. }\end{array}$ & $\begin{array}{l}\text { Stands } \\
\text { name }\end{array}$ & E & $\mathrm{N}$ & Alt \\
\hline & & 26 & $\begin{array}{l}\text { Abu } \\
\text { kasaba-2 }\end{array}$ & 33.88952 & 28.52474 & 1850 \\
\hline \multirow{2}{*}{$` 10$} & \multirow{2}{*}{$\begin{array}{l}\text { Abu } \\
\text { towatah }\end{array}$} & 27 & $\begin{array}{l}\text { Abu } \\
\text { towatah-1 }\end{array}$ & 33.89023 & 28.572827 & 1800 \\
\hline & & 28 & $\begin{array}{l}\text { Abu } \\
\text { towatah-2 }\end{array}$ & 33.89052 & 28.57717 & 1808 \\
\hline \multirow{2}{*}{11} & \multirow{2}{*}{ Abu walia } & 29 & $\begin{array}{l}\text { Abu walia- } \\
1\end{array}$ & 33.40772 & 28.53904 & 1921 \\
\hline & & 30 & $\begin{array}{l}\text { Abu walia- } \\
2\end{array}$ & 33.90899 & 28.53624 & 1902 \\
\hline \multirow{3}{*}{12} & \multirow{3}{*}{$\begin{array}{l}\text { Wadi al- } \\
\text { Talah }\end{array}$} & 31 & $\begin{array}{l}\text { Wadi al- } \\
\text { talah-1 }\end{array}$ & 33.9226 & 28.58395 & 1429 \\
\hline & & 32 & $\begin{array}{l}\begin{array}{l}\text { Wadi al- } \\
\text { talah-2 }\end{array} \\
\end{array}$ & 33:92565 & 28.57904 & 1451 \\
\hline & & 33 & $\begin{array}{l}\begin{array}{l}\text { Wadi al- } \\
\text { talah-3 }\end{array} \\
\end{array}$ & 33.93108 & 28.57148 & 1479 \\
\hline 13 & $\begin{array}{l}\text { Tunea } \\
\text { elkalabia }\end{array}$ & 34 & $\begin{array}{l}\text { Tunea } \\
\text { elkalabia }\end{array}$ & 33.90809 & 28.56388 & 1850 \\
\hline 14 & $\begin{array}{l}\text { Shak } \\
\text { tunea }\end{array}$ & 35 & Shak tunea & 33.90157 & 28.57951 & 1766 \\
\hline 15 & Tobok & 36 & Tobok & 33.93287 & 28.54076 & 1825 \\
\hline 16 & $\begin{array}{l}\begin{array}{l}\text { Shakef } \\
\text { tobok }\end{array} \\
\end{array}$ & 37 & $\begin{array}{l}\begin{array}{l}\text { Shakef } \\
\text { tobok }\end{array} \\
\end{array}$ & 33.93272 & 28.54271 & 1842 \\
\hline 17 & Taupq & 38 & Taupq & 33.87923 & $28: 55097$ & 1804 \\
\hline \multirow[b]{2}{*}{18} & \multirow[b]{2}{*}{ Meslh } & 39 & Mesalh & 33.88888- & 28.27527 & 1804 \\
\hline & & 40 & $\begin{array}{l}\text { Nakp } \\
\text { mesalh }\end{array}$ & 33.87785 & 28.56882 & 1853 \\
\hline 19 & $\begin{array}{l}\text { Shake } \\
\text { saker }\end{array}$ & 41 & Shake saker & 33.90013 & 28.57801 & 1779 \\
\hline 20 & Sakarkiah & 42 & Sakarkiah & 33.91119 & 28.55866 & 1540 \\
\hline 21 & $\begin{array}{l}\text { Naqb al- } \\
\text { zawateen }\end{array}$ & 43 & $\begin{array}{l}\text { Naqb al- } \\
\text { zawateen }\end{array}$ & 33.9338 & 28.54524 & 1882 \\
\hline 22 & Seleebat & 44 & Seleebat & 33.92359 & 28.54021 & 1882 \\
\hline
\end{tabular}


Table 2. Soil Physical properties of different Stands in SPK, south Sinai Egypt during June of 2014.

\begin{tabular}{|c|c|c|c|c|c|c|c|c|}
\hline \multirow{2}{*}{ No. } & \multirow{2}{*}{ Stand name } & \multicolumn{6}{|c|}{ Texture $\%$} & \multirow{2}{*}{ Texture } \\
\hline & & Water content & Fine grave & Coarse sand & fine sand & Silt & clay & \\
\hline 1 & Farsh al-lozaa-1 & 1.4 & 20 & 59 & 11 & 6 & 4 & loamy sand \\
\hline 2 & Farsh al-lozaa-2 & 1.09 & 37 & 50 & 5 & 4 & 4 & loamy sand \\
\hline 3 & Farsh al-lozaa-3 & 1.95 & 16 & 50 & 12 & 12 & 10 & sandy loam \\
\hline 4 & Farsh al-lozaa-4 & 1.2 & 31 & 46 & 7 & 10 & 6 & sandy loam \\
\hline 5 & Farsh al-sofsafa & 1.07 & 15 & 44 & 15 & 15 & 11 & sandy loam \\
\hline 6 & Farsh al-romana-1 & 1.7 & 2 & 88 & 8 & 1 & 1 & Sand \\
\hline 7 & Farsh al-romana-2 & 1.9 & 21 & 72 & 5 & 1 & 1 & Sand \\
\hline 8 & Graginiah-1 & 0.64 & 35 & 45 & 6 & 6 & 8 & sandy loam \\
\hline 9 & Graginiah-2 & 0.8 & 13 & 45 & 10 & 15 & 17 & sandy loam \\
\hline 10 & Graginiah-3 & 0.5 & 12 & 56 & 12 & 8 & 12 & sandy loam \\
\hline 11 & Shak muosa-1 & 1.7 & 26 & 55 & 8 & 5 & 6 & loamy sand \\
\hline 12 & Shak muosa-2 & 1.65 & 10 & 34 & 9 & 7 & 40 & sandy clay \\
\hline 13 & Wadi elarbain-1 & 1.54 & 23 & 45 & 10 & 12 & 10 & sandy loam \\
\hline 14 & Wadi elarbain-2 & 0.96 & 22 & 52 & 10 & 8 & 8 & sandy loam \\
\hline 15 & Wadi elarbain-3 & 1.8 & 15 & 50 & 12 & 12 & 11 & loamy sand \\
\hline 16 & Wadi al-shak-1 & 1.2 & 30 & 50 & 5 & 9 & 6 & sandy loam \\
\hline 17 & Wadi al-shak-2 & 1.32 & 30 & 54 & 7 & 6 & 3 & loamy sand \\
\hline 18 & Wadi al-shak-3 & 1.52 & 12 & 55 & 13 & 11 & 9 & sandy loam \\
\hline 19 & Wadi itlah-1 & 1.46 & 26 & 45 & 9 & 10 & 10 & sandy loam \\
\hline 20 & Wadi itlah-2 & 1.65 & 21 & 65 & 9 & 4 & 1 & Sand \\
\hline 21 & Wadi itlah-3 & 1.8 & 10 & 42 & 17 & 22 & 9 & sandy loam \\
\hline 22 & Maen al-raian-1 & 1.68 & 25 & 67 & 5 & 2 & 1 & Sand \\
\hline 23 & Maen al-raian-2 & 1.78 & 7 & 35 & 18 & 25 & 15 & sandy loam \\
\hline 24 & Maen al-raian-3 & 1.46 & 19 & 65 & 9 & 5 & 2 & Sand \\
\hline 25 & Abu kasaba-1 & 1.2 & 16 & 66 & 7 & 7 & 4 & loamy sand \\
\hline 26 & Abu kasaba-2 & 1.12 & 24 & 62 & 9 & 2 & 3 & Sand \\
\hline 27 & Abu towatah-1 & 1.42 & 25 & 50 & 11 & 9 & 5 & loamy sand \\
\hline 28 & Abu towatah-2 & 1.7 & 31 & 60 & 6 & 2 & 1 & Sand \\
\hline 29 & Abu walia-1 & 1.67 & 19 & 38 & 11 & 14 & 18 & sandy clay loama \\
\hline 30 & Abu walia-2 & 1.32 & 16 & 67 & 8 & 6 & 3 & Sand \\
\hline 31 & Wadi al-Talah-1 & 1.46 & 13 & 54 & 17 & 11 & 5 & loamy sand \\
\hline 32 & Wadi al-talah-2 & 1.6 & 22 & 48 & 11 & 12 & 7 & sandy loam \\
\hline 33 & Wadi al-talah-3 & 1.9 & 13 & 44 & 17 & 18 & 8 & sandy loam \\
\hline 34 & Tunea elkalabia & 1.14 & 24 & 62 & 6 & 5 & 3 & Sand \\
\hline 35 & Shak tunea & 1.3 & 15 & 60 & 13 & 5 & 7 & loamy sand \\
\hline 36 & Tobok & 0.5 & 35 & 44 & 8 & 7 & 6 & loamy sand \\
\hline 37 & Shakef tobok & 0.6 & 9 & 53 & 15 & 13 & 10 & sandy loam \\
\hline 38 & Taupq & 0.76 & 48 & 37 & 4 & 5 & 6 & sandy loam \\
\hline 39 & Shakef meslh & 1.82 & 42 & 54 & 2 & 1 & 1 & Sand \\
\hline 40 & Nakp mesalh & 0.8 & 13 & 48 & 17 & 16 & 6 & sandy loam \\
\hline 41 & Shakef saker & 1.42 & 16 & 67 & 9 & 5 & 3 & Sand \\
\hline 42 & Sakarkiah & 1.12 & 25 & 51 & 8 & 6 & 10 & sandy loam \\
\hline 43 & Naqb al-zawateen & 1.2 & 2 & 91 & 1 & 4 & 2 & Sand \\
\hline 44 & Seleebat & 0.64 & 16 & 59 & 12 & 7 & 6 & loamy sand \\
\hline \multicolumn{2}{|c|}{ LSD at $(0.05)$} & 0.17 & 6.87 & 6.87 & 3.42 & 2.98 & 2.90 & \\
\hline \multicolumn{2}{|c|}{ LSD at (0.01) } & 0.23 & 8.16 & 9.11 & 4.54 & 3.95 & 3.85 & \\
\hline
\end{tabular}


Table 3. Soil chemical properties of different stands in SPK, south Sinai Egypt during June of 2014.

\begin{tabular}{|c|c|c|c|c|c|c|c|c|c|c|c|c|c|}
\hline \multirow[b]{2}{*}{ No. } & \multirow[b]{2}{*}{ Stand name } & \multirow[b]{2}{*}{$\mathbf{p H}$} & \multirow[b]{2}{*}{$\begin{array}{l}\text { T.D.S } \\
\text { ppm }\end{array}$} & \multirow[b]{2}{*}{$\begin{array}{c}\mathrm{EC} \\
\mathrm{us} / \mathrm{cm}\end{array}$} & \multirow{2}{*}{$\begin{array}{c}\text { Org. } \\
\text { matter } \\
\%\end{array}$} & \multirow[b]{2}{*}{$\begin{array}{c}\mathrm{CaCo3} \\
\%\end{array}$} & \multicolumn{4}{|c|}{ Cations } & \multicolumn{3}{|c|}{ Anions (meq/L) } \\
\hline & & & & & & & $\underset{\text { meq/L }}{\mathbf{C a}^{++}}$ & $\underset{\text { meq/L }}{\mathbf{M g}^{++}}$ & $\begin{array}{l}\mathbf{N a}^{++} \\
\text {PPM }\end{array}$ & $\begin{array}{l}\mathbf{k}_{\mathbf{P P M}}^{+} \\
\end{array}$ & $\mathrm{HCO}_{3}{ }^{-}$ & $\mathrm{Cl}^{-}$ & $\mathrm{SO}_{4}^{-}$ \\
\hline 1 & Farsh al-lozaa-1 & 8.10 & 72.00 & 111.67 & 2.99 & 27.00 & 1.33 & 4.17 & 6.05 & 9.12 & 5.00 & 21.47 & 20.33 \\
\hline 2 & Farsh al-lozaa-2 & 7.87 & 113.00 & 176.67 & 1.84 & 23.17 & 2.33 & 5.50 & 6.80 & 5.70 & 5.00 & 37.33 & 41.00 \\
\hline 3 & Farsh al-lozaa-3 & 8.47 & 53.00 & 83.33 & 4.00 & 23.17 & 1.33 & 2.50 & 3.00 & 5.88 & 5.00 & 48.53 & 50.67 \\
\hline 4 & Farsh al-lozaa-4 & 8.70 & 69.00 & 106.67 & 3.27 & 25.50 & 1.33 & 4.83 & 4.63 & 6.68 & 5.00 & 24.73 & 77.67 \\
\hline 5 & Farsh al-sofsafa & 7.90 & 116.33 & 180.00 & 2.07 & 28.83 & 6.33 & 5.17 & 5.88 & 4.23 & 5.00 & 44.80 & 15.67 \\
\hline 6 & Farsh al-romana-1 & 8.73 & 67.67 & 103.33 & 7.91 & 25.33 & 2.33 & 0.17 & 3.38 & 8.00 & 5.00 & 27.53 & 47.00 \\
\hline 7 & Farsh al-romana-2 & 8.80 & 62.33 & 93.33 & 8.00 & 23.00 & 1.33 & 1.83 & 4.32 & 12.28 & 5.00 & 31.27 & 23.33 \\
\hline 8 & Graginiah-1 & 7.97 & 145.00 & 226.67 & 0.69 & 30.50 & 3.33 & 5.83 & 6.38 & 6.90 & 5.00 & 56.47 & 41.00 \\
\hline 9 & Graginiah-2 & 8.13 & 55.67 & 86.67 & 0.69 & 29.83 & 2.00 & 4.50 & 16.53 & 3.53 & 5.00 & 46.20 & 35.67 \\
\hline 10 & Graginiah-3 & 8.43 & 53.67 & 83.33 & 0.50 & 29.00 & 1.67 & 2.17 & 11.43 & 14.50 & 5.00 & 36.40 & 42.67 \\
\hline 11 & Shak muosa-1 & 8.07 & 120.00 & 186.67 & 4.30 & 28.17 & 2.33 & 6.17 & 6.13 & 4.50 & 5.00 & 48.07 & 60.67 \\
\hline 12 & Shak muosa-2 & 7.97 & 87.67 & 135.00 & 2.76 & 30.00 & 3.00 & 2.83 & 8.87 & 6.23 & 5.00 & 35.93 & 24.33 \\
\hline 13 & Wadi elarbain-1 & 7.73 & 136.67 & 210.00 & 3.45 & 31.00 & 3.67 & 8.17 & 12.40 & 12.58 & 5.00 & 18.67 & 31.00 \\
\hline 14 & Wadi elarbain-2 & 7.70 & 76.33 & 113.33 & 1.84 & 27.33 & 2.33 & 4.50 & 10.97 & 12.85 & 5.00 & 15.40 & 10.33 \\
\hline 15 & Wadi elarbain-3 & 7.73 & 73.33 & 106.67 & 4.37 & 29.33 & 3.33 & 6.83 & 7.38 & 6.92 & 5.00 & 19.13 & 11.33 \\
\hline 16 & Wadi al-shak-1 & 8.53 & 174.00 & 266.67 & 3.68 & 27.00 & 4.33 & 7.50 & 26.32 & 16.50 & 5.00 & 12.60 & 15.67 \\
\hline 17 & Wadi al-shak-2 & 7.73 & 516.67 & 800.00 & 3.22 & 31.83 & 8.67 & 16.50 & 25.58 & 37.40 & 5.00 & 13.53 & 71.00 \\
\hline 18 & Wadi al-shak-3 & 7.90 & 95.33 & 150.00 & 3.91 & 25.33 & 2.33 & 4.83 & 10.10 & 20.50 & 5.00 & 19.13 & 16.00 \\
\hline 19 & Wadi itlah-1 & 8.07 & 79.67 & 116.67 & 6.90 & 26.67 & 2.33 & 2.17 & 3.12 & 10.07 & 5.00 & 33.60 & 12.33 \\
\hline 20 & Wadi itlah-2 & 8.10 & 121.33 & 189.33 & 7.36 & 27.33 & 2.33 & 3.17 & 6.82 & 9.05 & 5.00 & 31.03 & 19.33 \\
\hline 21 & Wadi itlah-3 & 8.27 & 92.00 & 143.33 & 6.67 & 26.67 & 2.00 & 4.50 & 4.60 & 3.92 & 5.00 & 18.20 & 14.67 \\
\hline 22 & Maen al-raian-1 & 9.43 & 228.00 & 350.00 & 8.05 & 29.50 & 4.33 & 7.83 & 16.82 & 19.78 & 5.00 & 9.80 & 26.67 \\
\hline 23 & Maen al-raian-2 & 8.03 & 139.33 & 219.67 & 5.06 & 28.00 & 3.67 & 5.83 & 8.00 & 12.58 & 5.00 & 16.57 & 26.33 \\
\hline 24 & Maen al-raian-3 & 8.07 & 60.33 & 89.33 & 4.60 & 28.00 & 1.67 & 4.83 & 3.60 & 6.57 & 5.00 & 14.47 & 24.33 \\
\hline 25 & Abu kasaba-1 & 7.97 & 97.67 & 150.00 & 3.68 & 27.83 & 6.00 & 4.83 & 10.18 & 14.92 & 5.00 & 20.53 & 21.67 \\
\hline 26 & Abu kasaba-2 & 8.47 & 62.00 & 100.00 & 4.78 & 28.00 & 3.33 & 6.83 & 3.17 & 5.12 & 5.00 & 34.07 & 51.33 \\
\hline 27 & Abu towatah-1 & 8.37 & 45.67 & 66.67 & 10.58 & 30.00 & 2.00 & 2.83 & 3.33 & 3.32 & 5.00 & 17.27 & 15.00 \\
\hline 28 & Abu towatah-2 & 8.63 & 138.67 & 213.33 & 4.60 & 36.67 & 2.67 & 5.17 & 3.75 & 11.48 & 5.00 & 24.27 & 62.00 \\
\hline 29 & Abu walia-1 & 7.93 & 139.33 & 213.33 & 5.52 & 31.33 & 2.67 & 2.67 & 13.53 & 12.37 & 5.00 & 23.80 & 14.17 \\
\hline 30 & Abu walia-2 & 7.97 & 111.33 & 173.33 & 5.75 & 30.67 & 3.33 & 6.17 & 2.03 & 4.50 & 5.00 & 12.60 & 51.67 \\
\hline 31 & Wadi al-Talah-1 & 7.90 & 85.00 & 132.67 & 4.37 & 32.33 & 2.67 & 6.83 & 9.95 & 11.33 & 5.00 & 19.60 & 19.33 \\
\hline 32 & Wadi al-talah-2 & 8.07 & 74.00 & 106.67 & 5.06 & 28.00 & 2.00 & 2.50 & 5.00 & 7.25 & 5.00 & 21.47 & 19.33 \\
\hline
\end{tabular}




\begin{tabular}{|c|c|c|c|c|c|c|c|c|c|c|c|c|c|}
\hline \multirow[b]{2}{*}{ No. } & \multirow[b]{2}{*}{ Stand name } & \multirow[b]{2}{*}{$\mathbf{p H}$} & \multirow[b]{2}{*}{$\begin{array}{l}\text { T.D.S } \\
\text { ppm }\end{array}$} & \multirow[b]{2}{*}{$\begin{array}{c}\text { EC } \\
\text { us/cm }\end{array}$} & \multirow{2}{*}{$\begin{array}{c}\text { Org. } \\
\text { matter } \\
\%\end{array}$} & \multirow{2}{*}{$\begin{array}{c}\mathrm{CaCo3} \\
\%\end{array}$} & \multicolumn{4}{|c|}{ Cations } & \multicolumn{3}{|c|}{ Anions (meq/L) } \\
\hline & & & & & & & $\begin{array}{c}\mathrm{Ca}^{++} \\
\text {meq/L }\end{array}$ & $\underset{\text { meq/L }}{\mathbf{M g}^{++}}$ & $\begin{array}{l}\mathbf{N a}^{++} \\
\text {PPM }\end{array}$ & $\begin{array}{l}\mathbf{k}^{+} \\
\text {PPM }\end{array}$ & $\mathrm{HCO}_{3}{ }^{-}$ & $\mathrm{Cl}^{-}$ & $\mathrm{SO}_{4}{ }^{-}$ \\
\hline 33 & Wadi al-talah-3 & 8.20 & 72.00 & 110.00 & 8.51 & 29.67 & 3.00 & 3.83 & 6.22 & 5.47 & 5.00 & 24.27 & 18.00 \\
\hline 34 & Tunea elkalabia & 8.60 & 99.67 & 156.67 & 2.30 & 26.00 & 2.67 & 7.17 & 5.95 & 12.63 & 5.00 & 23.80 & 65.67 \\
\hline 35 & Shak tunea & 8.77 & 55.67 & 86.67 & 3.31 & 24.83 & 0.33 & 5.83 & 6.97 & 14.20 & 5.00 & 32.20 & 73.33 \\
\hline 36 & Tobok & 8.77 & 60.33 & 96.67 & 0.50 & 27.00 & 1.67 & 3.50 & 13.67 & 12.23 & 5.00 & 57.87 & 41.33 \\
\hline 37 & Shakef tobok & 8.30 & 77.67 & 120.00 & 1.38 & 29.17 & 1.33 & 3.17 & 9.22 & 3.85 & 5.00 & 41.53 & 50.00 \\
\hline 38 & Taupq & 7.97 & 74.67 & 110.00 & 0.92 & 31.25 & 2.83 & 3.33 & 6.17 & 5.62 & 5.00 & 53.67 & 13.33 \\
\hline 39 & Shakef meslh & 8.17 & 53.00 & 80.00 & 4.14 & 29.33 & 1.67 & 6.83 & 7.23 & 2.80 & 5.00 & 18.67 & 11.67 \\
\hline 40 & Nakp mesalh & 8.73 & 151.00 & 233.33 & 1.84 & 29.83 & 4.67 & 7.83 & 6.43 & 5.55 & 5.00 & 22.87 & 59.67 \\
\hline 41 & Shakef saker & 7.93 & 73.00 & 113.33 & 4.32 & 23.17 & 0.33 & 4.17 & 4.98 & 9.10 & 5.00 & 35.93 & 29.67 \\
\hline 42 & Sakarkiah & 8.30 & 73.67 & 116.67 & 3.63 & 24.33 & 3.00 & 3.17 & 10.63 & 4.90 & 5.00 & 44.33 & 56.00 \\
\hline 43 & Naqb al-zawateen & 8.23 & 78.00 & 120.00 & 4.14 & 25.67 & 2.33 & 8.50 & 11.98 & 8.05 & 5.00 & 41.53 & 70.00 \\
\hline 44 & Seleebat & 8.13 & 58.33 & 90.00 & 2.07 & 30.00 & 1.33 & 3.83 & 7.08 & 10.67 & 5.00 & 14.47 & 28.00 \\
\hline & LSD at $(0.05)$ & 0.26 & 26.18 & 41.11 & 1.81 & 3.90 & 1.36 & 1.98 & 1.98 & 2,38 & Ns & 6.78 & 5.14 \\
\hline & LSD at $(0.01)$ & 0.34 & 34.70 & 54.50 & 2.40 & 5.17 & 1.80 & 2.62 & 2.62 & 3.16 & Ns & 8.99 & 6.81 \\
\hline
\end{tabular}


Table 4. Essential oil percentage of O. syriacum plants in different stands in SPK, south Sinai Egypt during June of 2014.

\begin{tabular}{|c|l|c|c|l|c|}
\hline NO. & \multicolumn{1}{|c|}{ Stands } & $\begin{array}{c}\text { V. O } \\
\text { mll100g }\end{array}$ & NO. & \multicolumn{1}{c|}{ Stands } & $\begin{array}{c}\text { V. O } \\
\text { mll100g }\end{array}$ \\
\hline 1 & Farsh al-lozaa1 & 6.75 & 23 & Maen al-raian2 & 5.50 \\
\hline 2 & Farsh al-lozaa2 & 6.50 & 24 & Maen al-raian3 & 2.75 \\
\hline 3 & Farsh al-lozaa3 & 5.00 & 25 & Abu kasaba1 & 5.00 \\
\hline 4 & Farsh al-lozaa4 & 3.50 & 26 & Abu kasaba2 & 5.00 \\
\hline 5 & Farsh al-Sofsafa & 3.50 & 27 & Abu towatah1 & 4.50 \\
\hline 6 & Farsh al-romana1 & 4.00 & 28 & Abu towatah2 & 5.25 \\
\hline 7 & Farsh al-romana2 & 2.50 & 29 & Abu walia1 & 5.25 \\
\hline 8 & Graginiah 1 & 5.75 & 30 & Abu walia2 & 4.50 \\
\hline 9 & Graginiah 2 & 3.00 & 31 & Wadi al-Talah1 & 2.25 \\
\hline 10 & Graginiah 3 & 4.50 & 32 & Wadi al-Talah2 & 5.57 \\
\hline 11 & Shak mousa1 & 3.25 & 33 & Wadi al-Talah3 & 4.75 \\
\hline 12 & Shak mousa2 & 3.75 & 34 & Tunea elkalabia & 3.50 \\
\hline 13 & Wadi elarbain1 & 5.50 & 35 & Shak tunea & 5.00 \\
\hline 14 & Wadi elarbain2 & 5.25 & 36 & Tobok & 3.75 \\
\hline 15 & Wadi elarbain3 & 3.50 & 37 & Shakef tobok & 6.25 \\
\hline 16 & Wadi al-shak1 & 4.00 & 38 & Taupq & 4.50 \\
\hline 17 & Wadi al-shak2 & 3.25 & 39 & Shakef meslh & 4.00 \\
\hline 18 & Wadi al-shak3 & 3.50 & 40 & Nakp mesalh & 4.50 \\
\hline 19 & Wadi itlah1 & 5.75 & 41 & Shakef saker & 3.50 \\
\hline 20 & Wadi itlah 2 & 5.00 & 42 & Sakarkiah & 5.25 \\
\hline 21 & Wadi otlah3 & 5.00 & 43 & Naqb al-zawateen & 5.00 \\
\hline 22 & Maen al-raian1 & 3.50 & 44 & Seleebat & 6.00 \\
\hline
\end{tabular}


Table 5. The main constituents of essential oil of O. syriacum plants grown in stands in SPK, south Sinai Egypt during june of 2014

\begin{tabular}{|c|c|c|c|c|c|c|c|c|c|c|c|c|c|c|c|c|c|c|c|c|c|c|}
\hline \multirow{3}{*}{ Composite Name } & \multicolumn{22}{|c|}{ Stand No. } \\
\hline & 1 & 2 & 3 & 4 & 5 & 6 & 7 & 8 & 9 & 10 & 11 & 12 & 13 & 14 & 15 & 16 & 17 & 18 & 19 & 20 & 21 & 22 \\
\hline & A\% & A\% & A\% & A\% & A\% & $\mathbf{A \%}$ & A\% & A\% & A\% & A\% & A\% & A\% & A\% & A\% & A\% & A\% & A\% & A\% & A\% & A\% & A\% & A\% \\
\hline Thujene & 0.66 & 0.55 & 0.66 & 0.56 & 0.64 & 0.31 & 0.69 & 0.51 & 0.77 & 0.18 & 0.72 & 0.66 & 0.82 & 0.72 & 0.64 & 0.63 & 0.51 & 0.76 & 0.29 & 0.73 & 1.08 & 1.00 \\
\hline$\alpha$-pinen & 0.27 & 0.25 & 0.33 & 0.31 & 0.32 & 0.17 & 0.35 & 0.14 & 0.34 & 0.07 & 0.34 & 0.34 & 0.43 & 0.35 & 0.34 & 0.31 & 0.22 & 0.36 & 0.13 & 0.38 & 0.52 & 0.51 \\
\hline Comphere & 0.02 & 0.00 & 0.04 & 0.03 & 0.03 & 0.02 & 0.04 & 0.02 & 0.04 & 0.00 & 0.04 & 0.04 & 0.04 & 0.04 & 0.04 & 0.03 & 0.02 & 0.03 & 0.00 & 0.05 & 0.05 & 0.05 \\
\hline$\alpha$-myrcene & 0.02 & 0.05 & 0.01 & 0.01 & 0.00 & 0.00 & 0.02 & 0.01 & 0.02 & 0.00 & 0.02 & 0.00 & 0.02 & 0.00 & 0.02 & 0.00 & 0.00 & 0.02 & 0.03 & 0.02 & 0.02 & 0.00 \\
\hline Octanol & 0.02 & 0.00 & 0.03 & 0.03 & 0.00 & 0.01 & 0.04 & 0.00 & 0.04 & 0.00 & 0.00 & 0.00 & 0.00 & 0.00 & 0.00 & 0.00 & 0.05 & 0.00 & 0.03 & 0.06 & 0.01 & 0.00 \\
\hline$\alpha$-Tirpenene & 0.78 & 0.49 & 0.60 & 0.50 & 0.69 & 0.33 & 0.76 & 0.60 & 1.16 & 0.27 & 0.92 & 0.94 & 1.00 & 0.79 & 0.65 & 0.70 & 0.54 & 0.57 & 0.28 & 0.86 & 1.12 & 1.32 \\
\hline $\mathbf{P}$ - Cymene & 3.06 & 2.12 & 2.94 & 2.20 & 2.75 & 2.01 & 2.75 & 2.13 & 2.92 & 0.98 & 3.52 & 3.02 & 4.10 & 3.47 & 3.26 & 2.80 & 2.33 & 2.62 & 1.71 & 3.80 & 4.85 & 4.10 \\
\hline Di-limonene & 0.14 & 0.10 & 0.00 & 0.00 & 0.13 & 0.00 & 0.14 & 0.00 & 0.17 & 0.04 & 0.00 & 0.15 & 0.00 & 0.16 & 0.00 & 0.00 & 0.10 & 0.00 & 0.10 & 0.17 & 0.00 & 0.19 \\
\hline$\alpha$-phyllanderen & 0.11 & 0.10 & 0.09 & 0.10 & 0.09 & 0.06 & 0.10 & 0.11 & 0.12 & 0.03 & 0.12 & 0.12 & 0.11 & 0.14 & 0.11 & 0.08 & 0.08 & 0.07 & 0.05 & 0.14 & 0.15 & 0.15 \\
\hline $\mathrm{y}$-terpinene & 4.11 & 2.85 & 2.28 & 2.06 & 3.24 & 1.37 & 3.66 & 2.56 & 5.93 & 1.73 & 4.13 & 4.29 & 4.78 & 3.36 & 2.84 & 3.21 & 2.59 & 2.16 & 1.67 & 3.89 & 5.20 & 6.29 \\
\hline Trams - sabinen hydrate & 0.35 & 0.03 & 0.49 & 0.58 & 0.48 & 0.46 & 0.55 & 0.57 & 0.59 & 0.32 & 0.48 & 0.62 & 0.61 & 0.57 & 0.66 & 0.49 & 0.50 & 0.24 & 0.60 & 0.62 & 0.95 & 0.59 \\
\hline Linalool & 0.16 & 0.20 & 0.15 & 0.22 & 0.18 & 0.20 & 0.21 & 0.16 & 0.15 & 0.30 & 0.32 & 0.28 & 0.11 & 0.21 & 0.16 & 0.32 & 0.17 & 0.10 & 0.16 & 0.26 & 0.20 & 0.21 \\
\hline Borneol & 0.10 & 0.06 & 0.11 & 0.09 & 0.12 & 0.08 & 0.11 & 0.10 & 0.19 & 0.14 & 0.19 & 0.21 & 0.20 & 0.25 & 0.23 & 0.23 & 0.14 & 0.12 & 0.13 & 0.25 & 0.27 & 0.22 \\
\hline Terpieol -4 & 0.63 & 0.29 & 0.55 & 0.42 & 0.40 & 0.42 & 0.48 & 0.42 & 0.11 & 0.09 & 0.11 & 0.09 & 0.11 & 0.13 & 0.13 & 0.12 & 0.08 & 0.08 & 0.08 & 0.16 & 0.16 & 0.15 \\
\hline$\alpha$-Terpieol & 0.15 & 0.05 & 0.13 & 0.11 & 0.12 & 0.14 & 0.15 & 0.10 & 0.11 & 0.12 & 0.15 & 0.11 & 0.11 & 0.16 & 0.14 & 0.21 & 0.13 & 0.08 & 0.11 & 0.17 & 0.16 & 0.16 \\
\hline Carvone & 0.02 & 0.17 & 0.02 & 0.02 & 0.04 & 0.05 & 0.05 & 0.04 & 0.04 & 0.03 & 0.05 & 0.04 & 0.04 & 0.05 & 0.06 & 0.06 & 0.02 & 0.03 & 0.03 & 0.07 & 0.06 & 0.06 \\
\hline Thymol & 0.14 & 0.00 & 0.15 & 0.13 & 0.15 & 0.14 & 0.16 & 0.16 & 0.15 & 0.10 & 0.19 & 0.02 & 0.02 & 0.03 & 0.03 & 0.03 & 0.01 & 0.00 & 0.00 & 0.02 & 0.03 & 0.02 \\
\hline Carvacroal & 86.35 & 89.10 & 88.53 & 89.58 & 87.48 & 91.74 & 86.27 & 89.26 & 82.35 & 92.68 & 84.71 & 84.44 & \begin{tabular}{|l|}
83.54 \\
\end{tabular} & 85.08 & 86.59 & 86.53 & \begin{tabular}{|l|}
89.31 \\
\end{tabular} & \begin{tabular}{|l|}
90.03 \\
\end{tabular} & 91.41 & 83.66 & \begin{tabular}{|l|}
79.68 \\
\end{tabular} & 79.86 \\
\hline Euginol & 0.10 & 0.12 & 0.06 & 0.11 & 0.05 & 0.09 & 0.11 & 0.12 & 0.18 & 0.08 & 0.25 & \begin{tabular}{|l|}
0.09 \\
\end{tabular} & 0.07 & 0.08 & 0.11 & 0.21 & $\begin{array}{l}0.09 \\
\end{array}$ & \begin{tabular}{|l|}
0.05 \\
\end{tabular} & 0.11 & 0.16 & \begin{tabular}{|l|}
0.10 \\
\end{tabular} & 0.11 \\
\hline Long pinene epoxid & 0.53 & 0.69 & 0.52 & 0.63 & 0.70 & 0.64 & 0.69 & 0.82 & 1.62 & 0.75 & 0.89 & 1.35 & 0.84 & 0.88 & 0.82 & 0.77 & 0.57 & 0.38 & 1.00 & 0.86 & 0.87 & 0.94 \\
\hline $\begin{array}{l}\text { Total of oxyginated } \\
\text { compounds }\end{array}$ & 88.18 & 90.51 & 90.24 & 91.32 & 89.23 & 93.47 & 88.22 & 91.16 & 84.9 & 94.26 & 86.85 & 86.63 & 85.04 & 86.86 & 88.25 & 88.45 & 90.52 & \begin{tabular}{|l|}
90.87 \\
\end{tabular} & 93 & 85.59 & 81.52 & 81.72 \\
\hline $\begin{array}{l}\text { Total of non oxyginated } \\
\text { compounds }\end{array}$ & 9.54 & 6.71 & 7.45 & 6.37 & 8.38 & 4.77 & 9.11 & 6.67 & 12.1 & 3.65 & 10.3 & 10.18 & 11.91 & 9.61 & $\mathbf{8 . 5 8}$ & 8.28 & 6.94 & 6.83 & 4.92 & 10.74 & 13.96 & 14.21 \\
\hline Total & 97.72 & 97.22 & 97.69 & 97.69 & 97.61 & 98.24 & 97.33 & 97.83 & \begin{tabular}{|l|}
97.00 \\
\end{tabular} & 97.91 & 97.15 & 96.81 & 96.95 & 96.47 & 96.83 & 96.73 & 97.46 & \begin{tabular}{|l|}
97.70 \\
\end{tabular} & 97.92 & 96.33 & 95.48 & 95.93 \\
\hline
\end{tabular}


Table 5. cont.

\begin{tabular}{|c|c|c|c|c|c|c|c|c|c|c|c|c|c|c|c|c|c|c|c|c|c|c|}
\hline \multirow{3}{*}{ Composite Name } & \multicolumn{22}{|c|}{ Stand No. } \\
\hline & 23 & 24 & 25 & 26 & 27 & 28 & 29 & 30 & 31 & 32 & 33 & 34 & 35 & 36 & 37 & 38 & 39 & 40 & 41 & 42 & 43 & 44 \\
\hline & A\% & A\% & A\% & A\% & A\% & A\% & A\% & A\% & A\% & A\% & A\% & A\% & A\% & A\% & A\% & A\% & A\% & A\% & A\% & A\% & A\% & A\% \\
\hline Thujene & 0.74 & 0.17 & 0.84 & 0.91 & 1.06 & 0.75 & 0.43 & 0.80 & 0.84 & 0.87 & 0.49 & 0.75 & 0.79 & 0.64 & 0.28 & 0.85 & 0.91 & 0.52 & 0.20 & 0.38 & 1.03 & \begin{tabular}{|l|l|}
0.48 \\
\end{tabular} \\
\hline a-pinen & 0.36 & 0.00 & 0.39 & 0.43 & 0.51 & 0.40 & 0.20 & 0.41 & 0.39 & 0.42 & 0.00 & 0.45 & 0.39 & 0.39 & 0.17 & 0.50 & 0.41 & 0.28 & 0.14 & 0.23 & 0.52 & \begin{tabular}{|l|l|}
0.21 \\
\end{tabular} \\
\hline Comphore & 0.03 & 0.02 & 0.05 & 0.04 & 0.05 & 0.04 & 0.02 & 0.04 & 0.04 & 0.05 & 0.41 & 0.00 & 0.00 & 0.00 & 0.00 & 0.06 & 0.04 & 0.03 & 0.02 & 0.03 & \begin{tabular}{|l|}
0.06 \\
\end{tabular} & 0.03 \\
\hline$\alpha$-myrcene & 0.00 & 0.00 & 0.00 & 0.02 & 0.02 & 0.00 & 0.00 & 0.00 & 0.03 & 0.02 & 0.06 & 0.00 & 0.00 & 0.00 & 0.00 & 0.00 & 0.02 & 0.00 & 0.00 & 0.00 & \begin{tabular}{|l|}
0.02 \\
\end{tabular} & \begin{tabular}{|l|}
0.00 \\
\end{tabular} \\
\hline Octanol & 0.00 & 0.00 & 0.02 & 0.00 & 0.00 & 0.00 & 0.02 & 0.00 & 0.00 & 0.02 & 0.04 & 0.04 & 0.03 & 0.00 & 0.00 & 0.02 & 0.00 & 0.12 & 0.03 & 0.02 & \begin{tabular}{|l|l|}
0.00 \\
\end{tabular} & 0.00 \\
\hline$\alpha$-Tirpenene & 0.88 & 0.54 & 0.87 & 0.74 & 1.11 & 0.64 & 0.55 & 1.12 & 0.94 & 1.04 & 0.70 & 0.89 & 0.91 & 0.69 & 0.48 & 1.49 & 1.16 & 0.63 & \begin{tabular}{|l|l|}
0.59 \\
\end{tabular} & 0.61 & 1.08 & 0.71 \\
\hline $\mathbf{P}$-Cymene & 2.85 & 2.21 & 3.38 & 3.30 & 3.80 & 3.22 & 1.90 & $\mathbf{3 . 3 3}$ & 3.93 & 3.13 & 2.29 & 3.98 & 3.84 & 3.36 & 2.29 & 6.23 & 3.60 & 2.52 & \begin{tabular}{|l|l|}
4.40 \\
\end{tabular} & 3.16 & \begin{tabular}{|l|l|}
4.07 \\
\end{tabular} & \begin{tabular}{|l|}
3.31 \\
\end{tabular} \\
\hline Di -limonene & 0.14 & 0.00 & 0.17 & 0.00 & 0.18 & 0.00 & 0.00 & 0.16 & 0.00 & 0.17 & 0.16 & 0.00 & 0.19 & 0.00 & 0.00 & 0.26 & 0.18 & 0.00 & \begin{tabular}{|l|}
0.00 \\
\end{tabular} & 0.00 & 0.19 & 0.00 \\
\hline a-phyllanderen & 0.10 & 0.07 & 0.12 & 0.12 & 0.17 & 0.13 & 0.06 & 0.13 & 0.15 & 0.12 & 0.08 & 0.12 & 0.15 & 0.10 & 0.05 & 0.23 & 0.15 & 0.09 & \begin{tabular}{|l|l|}
0.07 & \\
\end{tabular} & 0.07 & 0.15 & \begin{tabular}{|l|l|}
0.09 \\
\end{tabular} \\
\hline$\gamma$-Terpinene & 4.47 & 2.73 & 4.08 & 2.93 & 5.05 & 2.30 & 2.75 & $\mathbf{5 . 3 0}$ & 4.09 & 5.06 & 3.09 & 3.95 & 4.09 & 2.75 & 2.74 & 7.40 & 5.41 & 2.61 & 2.67 & 3.08 & 4.64 & 3.35 \\
\hline Trams - sabinen hydrate & 0.73 & 0.50 & 0.69 & 0.61 & 0.65 & 0.53 & 0.68 & 0.82 & 0.44 & 0.53 & 0.49 & 1.20 & 0.73 & 0.67 & 0.74 & 1.32 & 0.73 & \begin{tabular}{|l|}
0.78 \\
\end{tabular} & \begin{tabular}{|l|}
0.92 \\
\end{tabular} & 0.76 & \begin{tabular}{|l|}
0.58 \\
\end{tabular} & \begin{tabular}{|l|l|}
0.56 \\
\end{tabular} \\
\hline Linalool & 0.16 & 0.16 & 0.28 & 0.22 & 1.84 & 0.25 & 0.18 & 0.25 & 0.29 & 0.31 & 0.09 & 0.16 & 0.20 & 0.11 & 0.00 & 0.31 & \begin{tabular}{|l|l|}
0.18 \\
\end{tabular} & \begin{tabular}{|l|}
0.29 \\
\end{tabular} & \begin{tabular}{|l|}
0.22 \\
\end{tabular} & 0.28 & \begin{tabular}{|l|}
0.25 \\
\end{tabular} & \begin{tabular}{|l|}
0.29 \\
\end{tabular} \\
\hline Borneol & 0.27 & 0.26 & 0.25 & 0.22 & 0.00 & 0.32 & 0.22 & 0.30 & 0.22 & 0.20 & 0.18 & 0.41 & 0.24 & 0.27 & \begin{tabular}{|l|}
0.22 \\
\end{tabular} & 0.42 & 0.27 & \begin{tabular}{|l|}
0.29 \\
\end{tabular} & \begin{tabular}{|l|}
0.42 \\
\end{tabular} & 0.37 & 0.26 & \begin{tabular}{|l|}
0.25 \\
\end{tabular} \\
\hline Terpienol -4 & 0.17 & 0.12 & 0.22 & 0.11 & 0.12 & 0.12 & 0.14 & 0.16 & 0.14 & 0.16 & 0.12 & 0.16 & 0.13 & 0.14 & 0.16 & 0.32 & 0.17 & 0.16 & 0.16 & 0.13 & \begin{tabular}{|l|}
0.17 \\
\end{tabular} & \begin{tabular}{|l|}
0.15 \\
\end{tabular} \\
\hline$\alpha$-Terpineol & 0.14 & 0.11 & 0.15 & 0.14 & 0.11 & 0.15 & 0.17 & 0.15 & 0.17 & 0.16 & 0.12 & 0.18 & 0.12 & 0.11 & \begin{tabular}{|l|}
0.07 \\
\end{tabular} & \begin{tabular}{|l|}
0.18 \\
\end{tabular} & 0.18 & \begin{tabular}{|l|l|}
0.13 \\
\end{tabular} & \begin{tabular}{|l|}
0.15 \\
\end{tabular} & 0.21 & \begin{tabular}{|l|}
0.16 \\
\end{tabular} & \begin{tabular}{|l|}
0.21 \\
\end{tabular} \\
\hline Carvone & 0.07 & 0.05 & 0.08 & 0.06 & 0.05 & 0.06 & 0.07 & 0.06 & 0.05 & 0.05 & 0.05 & 0.09 & 0.06 & 0.05 & \begin{tabular}{|l|}
0.00 \\
\end{tabular} & 0.07 & \begin{tabular}{|l|}
0.06 \\
\end{tabular} & \begin{tabular}{|l|}
0.06 \\
\end{tabular} & \begin{tabular}{|l|}
0.08 \\
\end{tabular} & 0.06 & \begin{tabular}{|l|l|}
0.07 \\
\end{tabular} & \begin{tabular}{|l|}
0.07 \\
\end{tabular} \\
\hline Thymol & 0.02 & 0.01 & 0.03 & 0.02 & 0.01 & 0.02 & 0.04 & 0.02 & 0.03 & 0.02 & 0.00 & 0.03 & 0.04 & 0.01 & \begin{tabular}{|l|}
0.00 \\
\end{tabular} & 0.03 & 0.02 & 0.05 & 0.03 & 0.03 & \begin{tabular}{|l|}
0.02 \\
\end{tabular} & \begin{tabular}{|l|l|}
0.03 \\
\end{tabular} \\
\hline Carvacroal & 84.05 & 89.34 & 83.68 & 85.83 & 80.66 & 86.86 & 88.01 & 82.17 & 83.38 & 83.13 & 85.60 & 82.78 & 83.55 & 86.99 & 90.22 & 74.21 & 81.49 & 87.39 & \begin{tabular}{|l|}
85.78 \\
\end{tabular} & 86.41 & 81.60 & 85.63 \\
\hline Euginol & 0.14 & 0.08 & 0.09 & 0.11 & 0.11 & 0.10 & 0.13 & 0.09 & \begin{tabular}{|l|}
0.14 \\
\end{tabular} & 0.05 & 0.10 & 0.16 & 0.13 & 0.05 & 0.08 & 0.10 & 0.10 & 0.08 & 0.16 & \begin{tabular}{|l|}
0.12 \\
\end{tabular} & \begin{tabular}{|l|}
0.12 \\
\end{tabular} & \begin{tabular}{|l|}
0.13 \\
\end{tabular} \\
\hline Long pinene epoxid & 1.28 & 0.89 & 0.92 & 0.82 & 0.72 & 0.93 & 1.34 & 0.88 & 1.02 & 0.88 & 0.67 & 0.77 & 0.97 & 0.56 & 0.44 & 1.02 & 0.70 & 0.86 & 0.98 & 0.79 & 0.96 & \begin{tabular}{|l|l|}
0.89 \\
\end{tabular} \\
\hline $\begin{array}{l}\text { Total of oxyginated } \\
\text { compounds }\end{array}$ & 86.26 & 90.99 & 5.67 & 87.51 & 83.62 & 99 & 5 & .06 & .43 & 84.96 & .29 & 84.65 & 85.38 & 88.24 & 91.19 & 76.65 & 3.15 & 9.28 & 7.92 & 8.37 & 3.6 & \begin{tabular}{|l|}
87.61 \\
\end{tabular} \\
\hline $\begin{array}{l}\text { Total of non oxyginated } \\
\text { compounds }\end{array}$ & 10.34 & 6.27 & 10.64 & 9 & 12.6 & 8 & 6 & 1 & 10.86 & 11.43 & 7.45 & 17 & 18 & 8.65 & 6.75 & 37 & 63 & 61 & 9.1 & 37 & 2.35 & 78 \\
\hline Total & 6.60 & 97.26 & 96.31 & 6.63 & 96.22 & \begin{tabular}{|l|l|}
96.82 \\
\end{tabular} & \begin{tabular}{|l|}
96.91 \\
\end{tabular} & 96.19 & \begin{tabular}{|l|}
96.29 \\
\end{tabular} & 96.39 & 94.74 & 96.12 & 96.56 & $\begin{array}{l}96.89 \\
\end{array}$ & 97.94 & 95.02 & 95.78 & $\begin{array}{l}96.89 \\
\end{array}$ & 97.02 & \begin{tabular}{|c|}
96.74 \\
\end{tabular} & \begin{tabular}{|l}
95.95 \\
\end{tabular} & \begin{tabular}{|l|}
96.39 \\
\end{tabular} \\
\hline
\end{tabular}




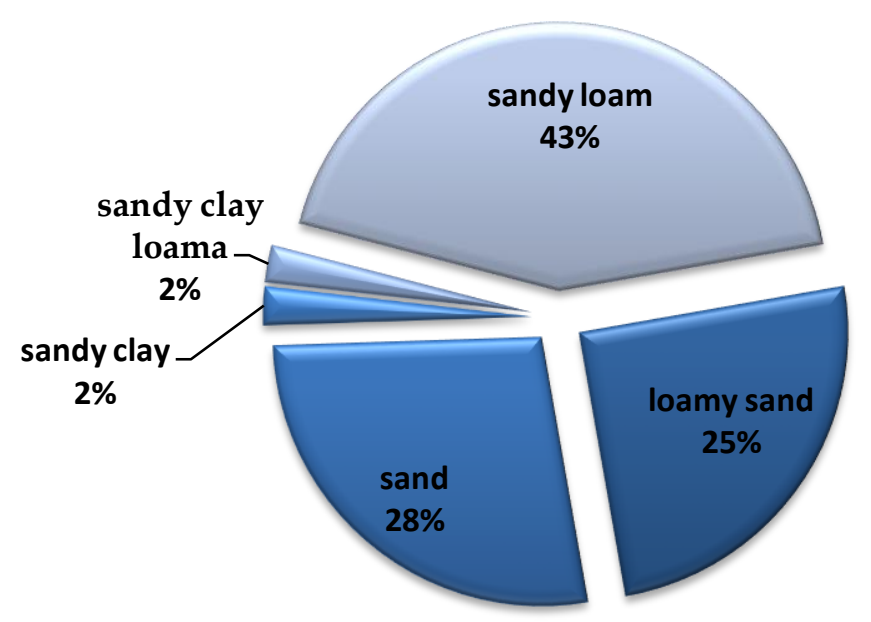

Fig.1. Texture types of soil and their percentages.

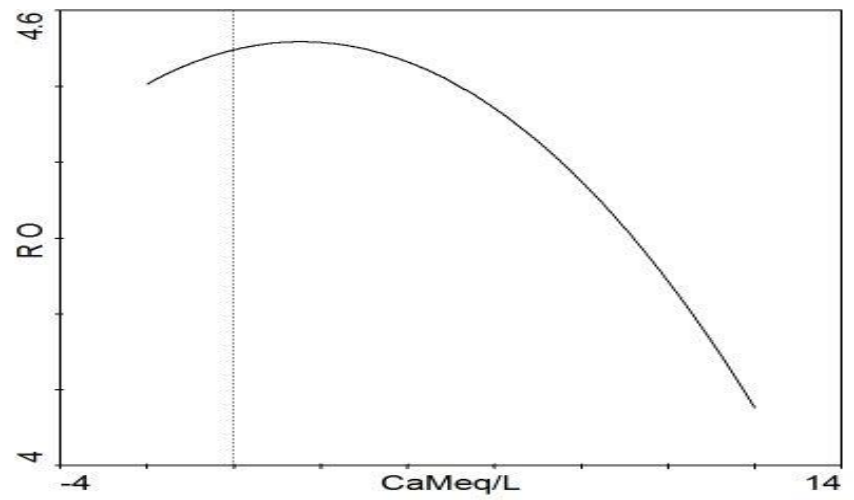

Fig. 1a. Relation between essential oil percentages of $O$. syriacum and calcium content in the soil.

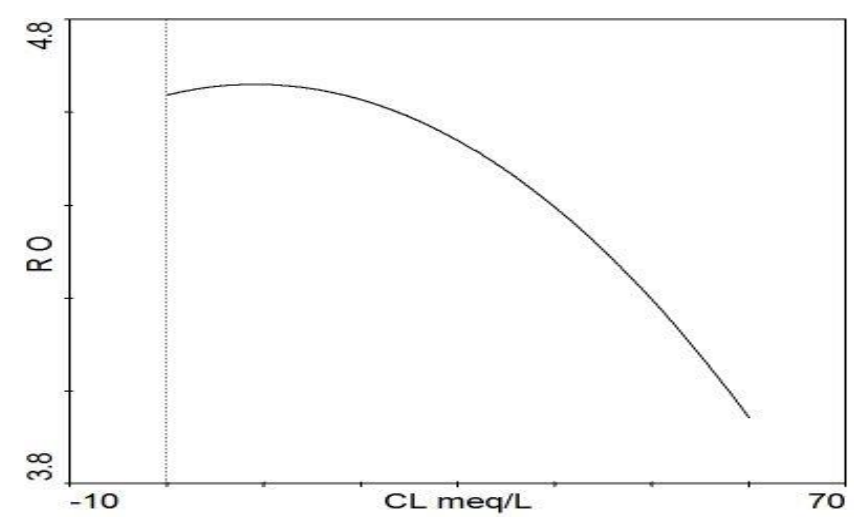

Fig. 2a. Relation between essential oil percentages of $O$. syriacum and chloride content in the soil.

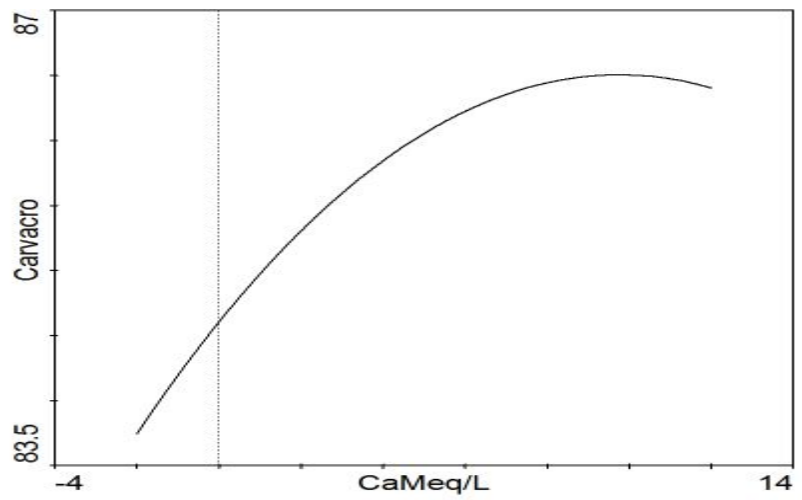

Fig. 1b. Relation between carvacrol content (\%) of $O$. Syriacum essential oil and calcium content in the soil.

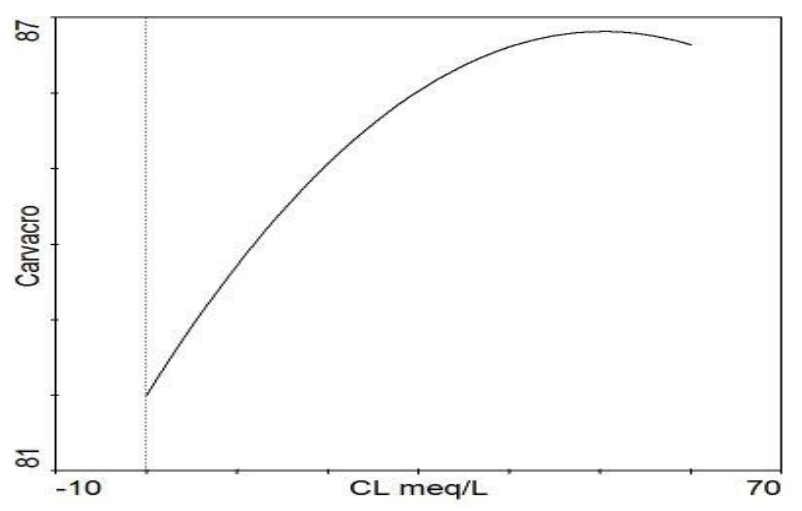

Fig. 2b: Relation between carvacrol content (\%) in the essential oil of $O$. syriacum and chloride content in the soil. 


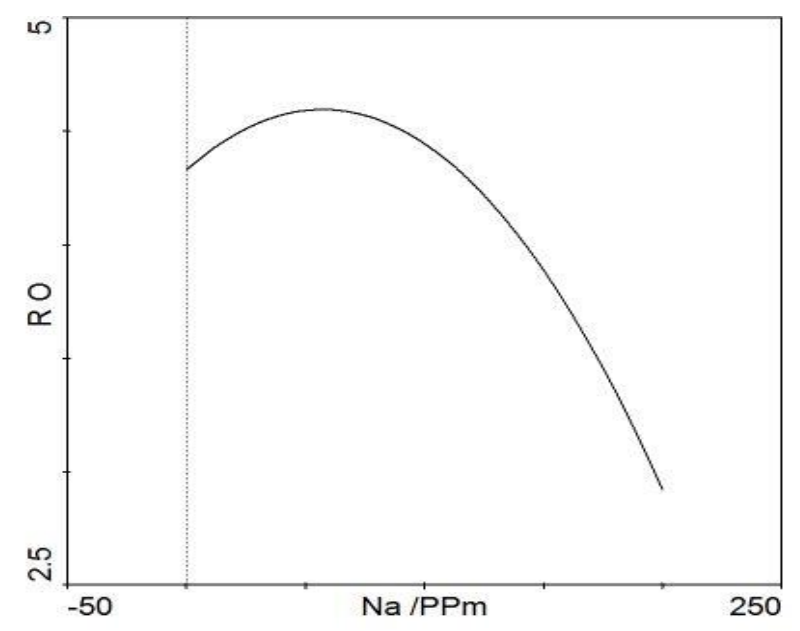

Fig. 3a. Relation between essential oil percentages of $O$. syriacum and sodium content in different stands.

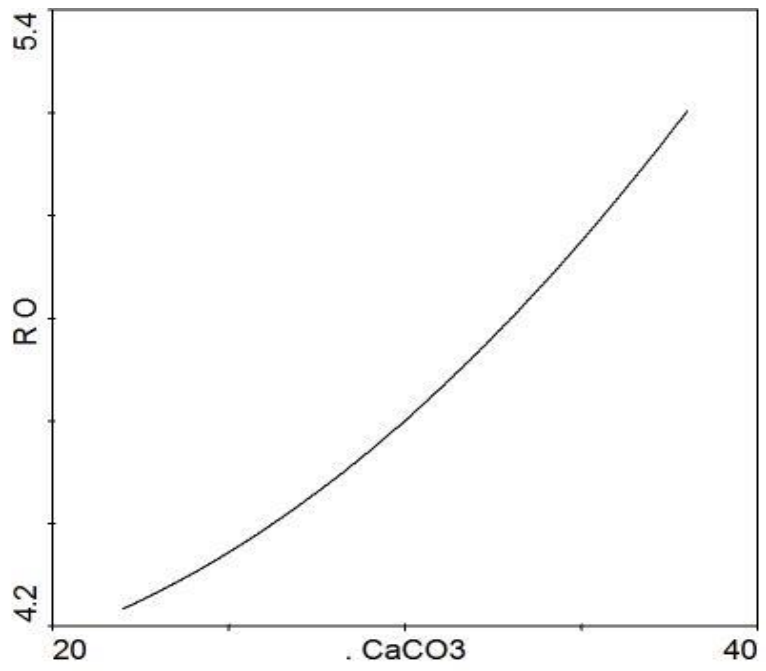

Fig. 4a. Relation between essential oil percentages of $O$. syriacum and calcium carbonate in different stands.

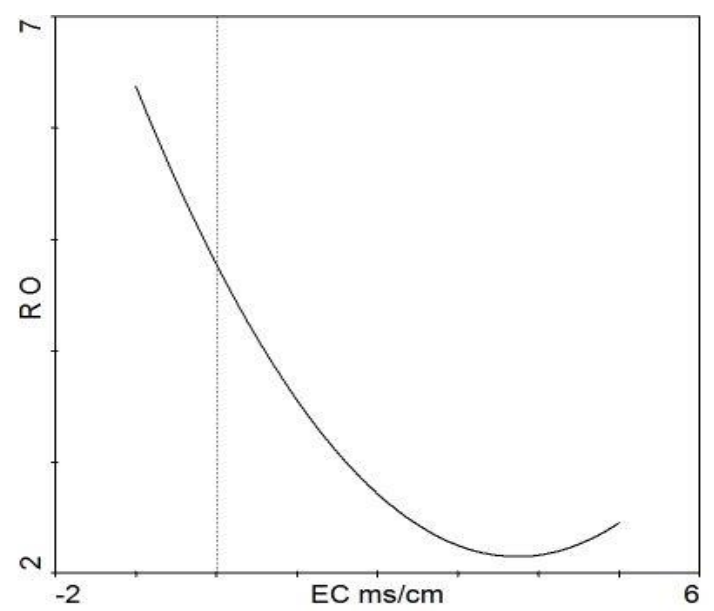

Fig. (5a). Relation between essential oil percentages of $O$. syriacum and electrical conductivity in different stands.

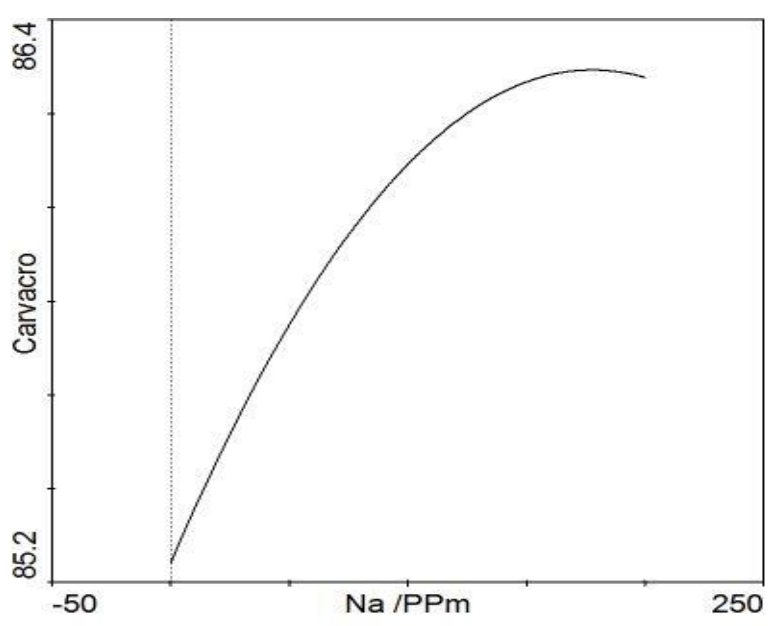

Fig. 3b. Relation between carvacrol content (\%) of $O$. syriacum and sodium content.in different stands.

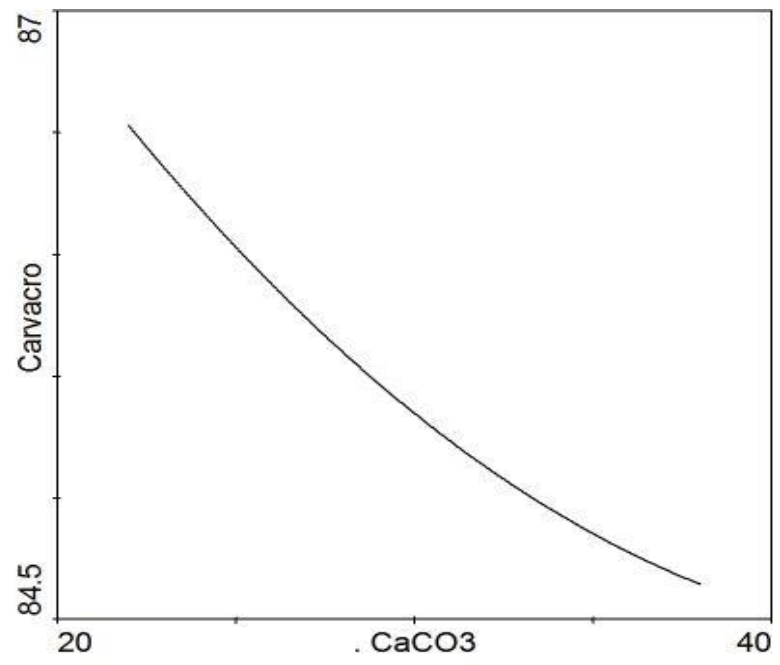

Fig. 4b. Relation between carvacrol content (\%) of $O$. syriacum and calcium carbonate in different stands.

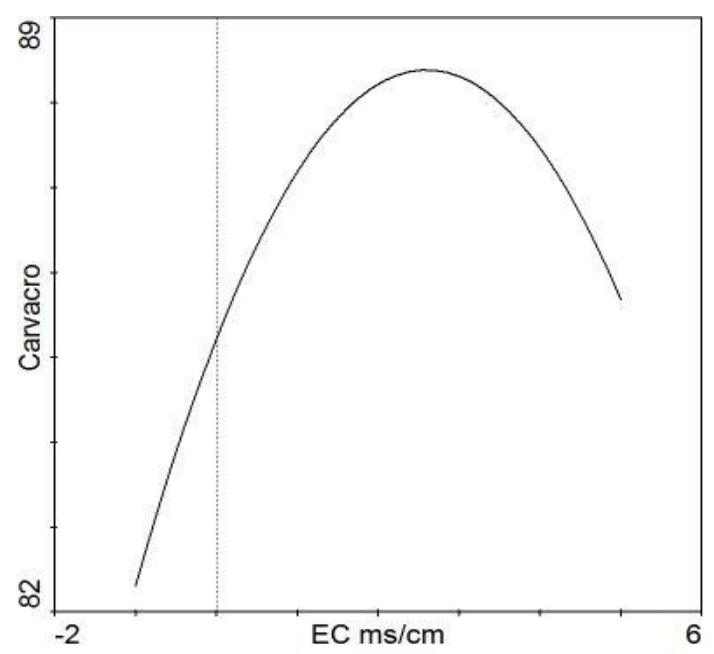

Fig. (5b). Relation between carvacrol content $(\%)$ of $O$. syriacum and the electrical conductivity in different stands. 


\section{المكونات الأساسية للزيت الطيار لزعتر كاتزين الناي برياً بمحمية كترين بجنوب سيناء مصر}

أنس عبد العزيز حسين 1"، أحمد محد عبد الغني 1، محسن أحمد عثمان المهندس 1، البيلي السيد أحمد حطب 2، السيد أبو الفتوح عمر 3

$$
\begin{aligned}
& \text { 1 قسم البيئة والوزراعة الحيوية، كلية الزراعة، جامعة المزهر، بالقاهة، مصر } \\
& \text { 2جماز شئون البيئة، وزارة الزراعة، القاهة، مصر }
\end{aligned}
$$

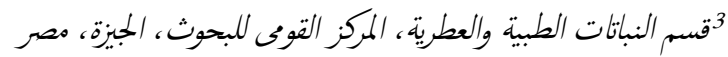

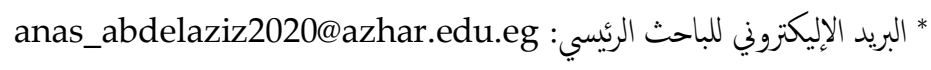

الملخص العربي

تهدف هذه الدراسة إلى دراسة تركيب زيت نبات زعتر كاترين الناي في مواقع ختنافة في سانت كاترين. تم تجميع النباتات برئاً بمحمية سانت كاترين التابعة لمحافظة

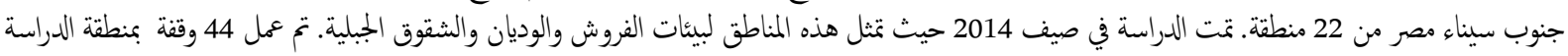

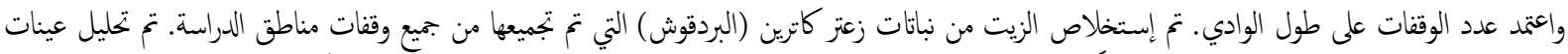

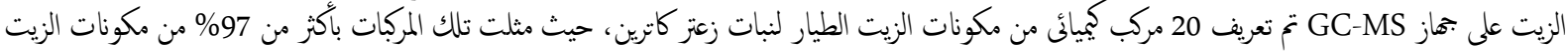

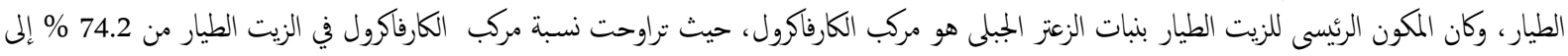

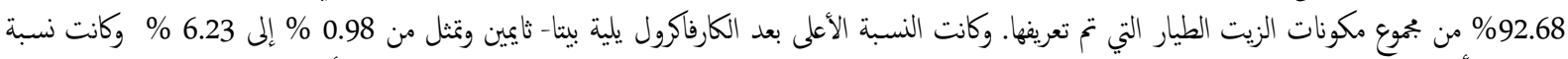

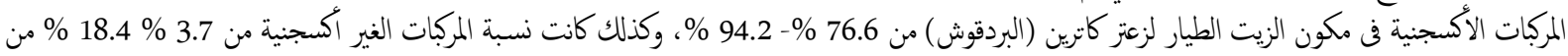

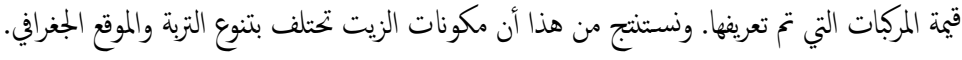
الكلمات المفتاحية: زعتر كاثرين، الزيت الطيار ، (GC-MS)، الكارفاكوول، سانت كانزين. 